\title{
Stable expression plasmids for Streptomyces based on a toxin-antitoxin system
}

\author{
Laura Sevillano, Margarita Díaz and Ramón I Santamaría
}

\begin{abstract}
Background: Bacteria included in the genus Streptomyces exhibit several attractive characteristics that make them adequate hosts for the heterologous expression of proteins. One of them is that some of its species have a high secretion capacity and hence the protein of interest could be released to the culture supernatant, facilitating downstream processing. To date, all the expression vectors described for these bacteria contain antibiotic resistance genes as selection markers. However, the use of antibiotics to produce proteins at industrial level is currently becoming more restricted owing to the possibility of contamination of the final product. In this report, we describe the use of the $S$. lividans yefM/yoeBs/ toxin-antitoxin system to develop a stable plasmid expression system.

Results: In order to use the yefM/yoeBs/ system to stabilize expression plasmids in Streptomyces, a S. lividans mutant strain that contained only the toxin gene (yoeBsl) in its genome and the antitoxin gene (yefMsl) located in a temperature-sensitive plasmid was constructed and used as host. This strain was transformed with an expression plasmid harbouring both the antitoxin gene and the gene encoding the protein of interest. Thus, after elimination of the temperature-sensitive plasmid, only cells with the expression plasmid were able to survive. On using this system, two proteins - an a-amylase from S. griseus and a xylanase from S. halstedii - were overproduced without the addition of antibiotic to the culture medium. The production of both proteins was high, even after long incubations ( 8 days), and after serial subcultures, confirming the stability of the plasmids without antibiotic selection.

Conclusions: This is the first report that describes the use of a toxin-antitoxin system to maintain high -copy plasmids in Streptomyces. This finding could be a valuable tool for using Streptomyces as a host to produce proteins at the industrial and pharmaceutical levels without the use of antibiotics in the production step.
\end{abstract}

Keywords: Streptomyces, Toxin-antitoxin, Recombinant protein, Plasmid stabilization

\section{Background}

The production of high levels of proteins for different purposes (scientific, therapeutic, diagnostic, environmental, agricultural, etc.) is one of the main goals of biotechnology and the choice of a suitable expression system is a crucial step in this process. Owing to its fast proliferation, high expression levels and short fermentation time, Escherichia coli is the host bacterium most commonly used for the large-scale production of recombinant proteins [1]. However, E. coli is not always suitable for the production of active proteins on account of problems of insolubility, cytotoxicity, inefficient translation or the inability to carry

\footnotetext{
* Correspondence: santa@usal.es

Instituto de Biología Funcional y Genómica. Consejo Superior de Investigaciones Científicas/Universidad de Salamanca, C/ Zacarías González n², Salamanca 37007, Spain
}

out post-translational modifications [2]. In order to overcome these problems, other prokaryotic and eukaryotic hosts have been used. Bacillus spp and Lactococcus are Gram-positive bacteria that are often used to secrete proteins into the culture medium [3-8]. Eukaryotic cells, such as yeast cells, insect cells or immortalized cell lines, are mainly used to produce active proteins with posttranslational modifications $[9,10]$.

Streptomyces is a promising bacterial expression system that has been used to produce high levels of several proteins $[1,11]$. Streptomycetes are aerobic, filamentous Gram-positive soil bacteria that secrete a wide range of extracellular enzymes to degrade a broad variety of substrates in order to survive.

As a host, Streptomyces has the following advantages over other systems: 1) the formation of inclusion bodies

\section{Biomed Central}


has not been described in the literature; 2) it is a wellsuited host for the expression of very GC-rich genes without codon adaptation [12]; 3) it has high protein secretion efficiency, which makes it feasible to obtain the protein of interest in the culture supernatant, facilitating the protein folding and downstream procedures of extraction and purification [11];4) the species used to express proteins (see below) display a relatively low level of endogenous extracellular proteolytic activity in comparison with other hosts such as Bacillus, where deletion of up to seven extracellular proteases had to be done to improve protein production $[13,14]$.
Among Streptomyces, S. lividans is the preferred host for producing recombinant proteins because it is genetically well characterized, it has low proteolytic activity and it lacks the restriction systems present in other Streptomyces species such as S. coelicolor. Accordingly, S. lividans can be directly transformed with $E$. coli-derived shuttle vectors. In fact, it has been used to produce a large number of proteins of both prokaryotic and eukaryotic origin. Most of them are summarized in two reviews $[1,11]$.

Our group has developed an expression system for Streptomyces based on the use of strong promoter such as the xysAp promoter from $S$. halstedii [15] regulated by

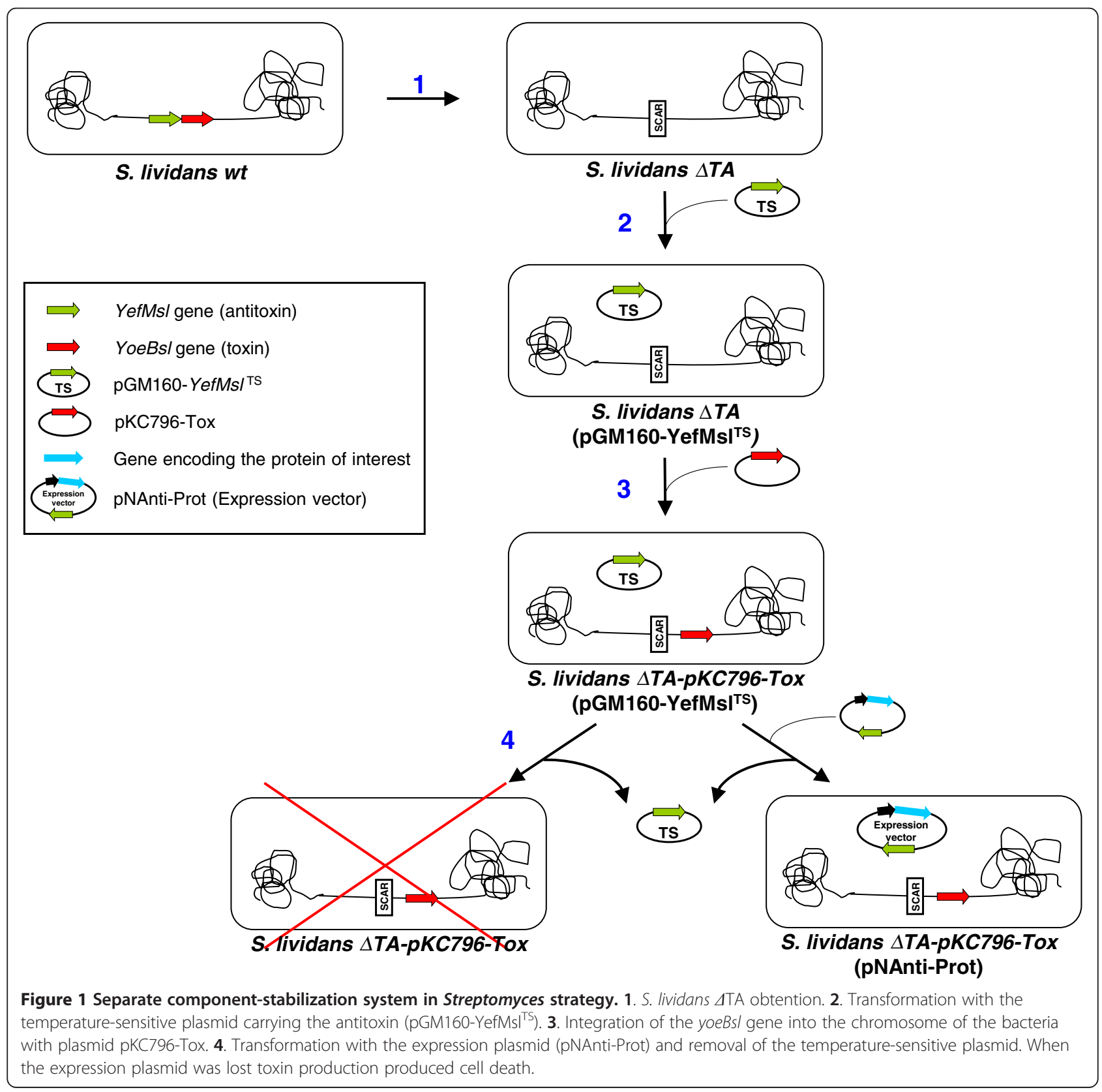


different carbon sources. The system has proved useful for the expression of genes from different Streptomyces species, from Aspergillus nidulans and from Thermus thermophilus $([12,16])$. This system uses multi-copy monofunctional (Streptomyces) or bi-functional ( $E$. coli and Streptomyces) plasmids that are selected with antibiotic resistance genes (mainly thiostrepton or neomycin). However, the use of antibiotics at large-scale entails several problems associated with the potential risk of contamination of the product, apart from its high cost. Moreover, the use of antibiotics in intensive culture conditions is not efficient owing to dilution or antibiotic inactivation $[17,18]$. This has made it necessary to develop alternative methods to stabilize plasmid-bearing cultures without adding antibiotics.

One alternative strategy is the use of toxin-antitoxin (TA) systems that involve host death upon plasmid loss. TA systems are widespread among the plasmids and genomes of bacteria and archaea and play important roles in responses to environmental stress as well as in the stability of mobile genetic material [19]. Class II TA systems consist of a biologically active protein molecule (toxin) and the corresponding inhibitor protein (antitoxin). The efficiency of these TA systems depends on the different lifespan of both proteins; while toxins are highly resistant to proteases, antitoxins have a very short half-life owing to their high susceptibility to protease activity. Toxin activity places cells in a dormant state, leading to cell death during prolonged exposure. TA systems have been used for the positive selection of transformants in $E$. coli $[17,18,20]$. Initially, the sequence encoding the full operon was included in the expression plasmid and in plasmid-free cells the antitoxin was degraded, leaving the toxin free to exert its effect and causing cell death [21]. However, this system can only delay the takeover of the culture by plasmid-free cells because the dilution of the toxin, as a consequence of bacterial growth, allows plasmid-free cells to survive. To surmount these problems, other systems have been developed based on the separation of the two components of the operon $[17,18]$. In these separate-component-stabilization (SCS) systems, the antitoxin is localized in the plasmid and the toxin is integrated in the bacterial chromosome. Thus, when the plasmid is present in the cell, the antitoxin counteracts the effect of the toxin, whereas upon plasmid loss the toxin causes cell death.

Some of these systems based on the separation of the two components of the operon are available commercially for E. coli (StabyCloning ${ }^{\mathrm{TM}}$ and StabyExpress ${ }^{\mathrm{TM}}$, Delphi Genetics $\mathrm{SA})$. In these, the toxin gene $(c c d B)$ is integrated into the host chromosome and the antitoxin gene $(\operatorname{ccd} A)$ is inserted

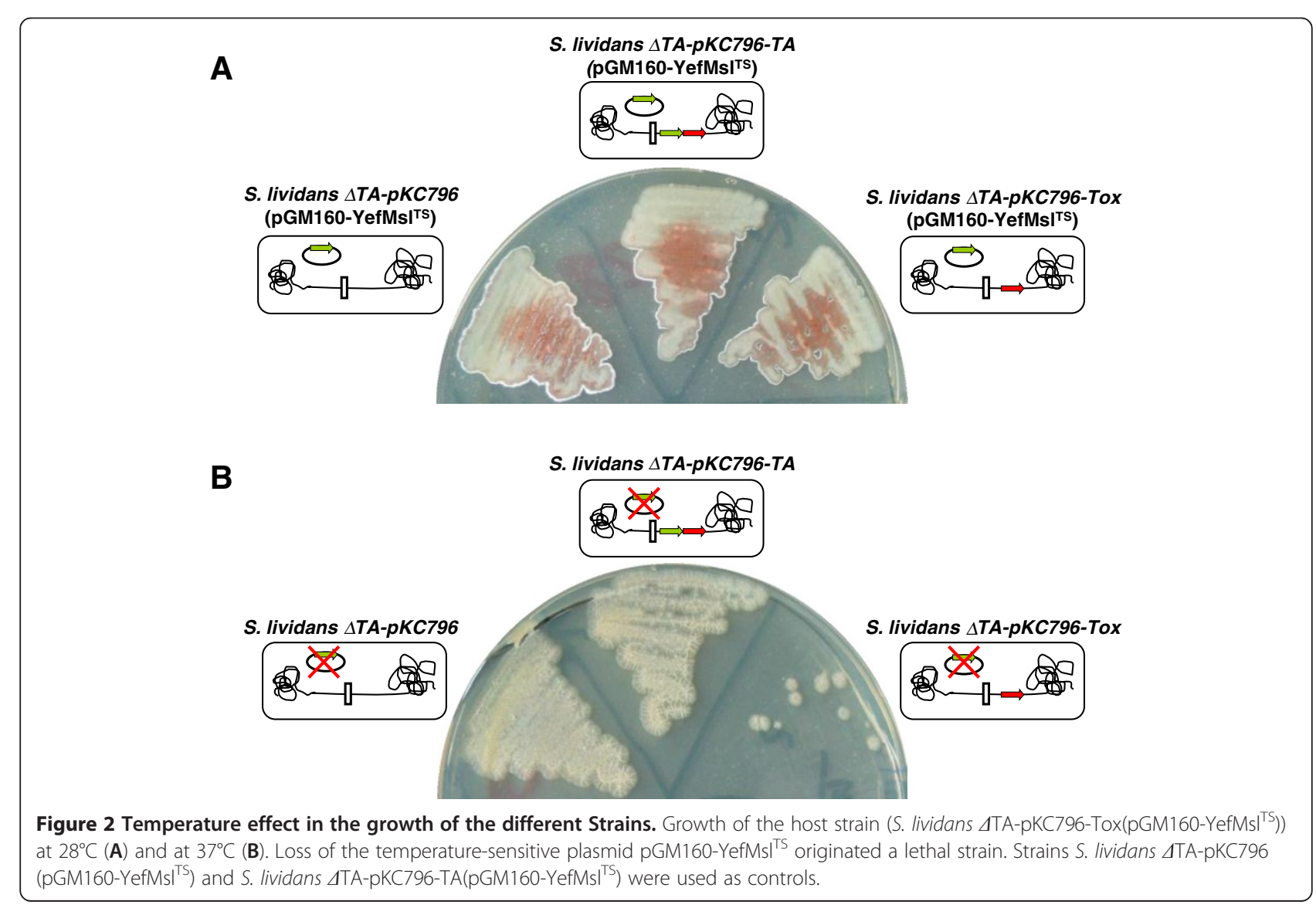


into an expression plasmid, and hence loss of the expression plasmid leads to cell death. They have been shown to be effective for plasmid stabilization without the use of antibiotics during the production process $[17,18,20]$.

By means of bioinformatics tools, up to 24 putative TA systems have been identified in the $S$. coelicolor genome (TADB) [22]. Our group has recently characterized the first toxin/antitoxin system from Streptomyces experimentally [23]. This system, from S. lividans, was named yefM/yoeBsl and is identical to the system encoded by SCO2235/ SCO2236 from S. coelicolor (yefM/yoeBsc). In this system, the toxin (YoeBsl) acts by inhibiting translation initiation due to its mRNA interferase activity [23].

Here, we describe a new strategy for the stabilization of expression plasmids in Streptomyces based on the use of the $y e f M / y o e B s l$ system as a selection marker. In a first step, we constructed the $S$. lividans host strain that contained only the toxin gene (yoeBsl) in the chromosome, while the antitoxin gene (yefMsl) was located in a temperaturesensitive plasmid. This strain was used as a host to produce proteins from an expression plasmid that contained both the antitoxin gene and the gene of the protein of interest. Thus, after removal of the temperature-sensitive plasmid containing the antitoxin gene, the loss of the expression plasmid led to cell death. This system was used to produce two proteins without the use of antibiotics during the production process. The production of both proteins remained high even in long-term and serial cultures. These results confirm the efficiency of the selection method as well as the stability of the expression plasmids.

\section{Results and discussion}

\section{Strategy}

In the present work we developed a Streptomyces separatecomponent-stabilization system with the yefM/yoeBsl TA genes from S. lividans [23]. The strategy followed during the work is summarized in Figure 1. In an initial step, we deleted the complete yefM/yoeBsl operon from the genome to obtain the $S$. lividans $\triangle T A$ strain (Figure 1 step 1); the integration of yoeBsl into the chromosome of the $\triangle T A$ strain was lethal [23]. Therefore, before integration of the toxin we needed to transform this strain with an easily removable plasmid containing the antitoxin. Thus, the $S$. lividans- $\triangle T A$ strain was transformed with a temperaturesensitive plasmid (pGM160 derivate [24]) containing the antitoxin gene (Figure 1 step 2). Then, the toxin gene was integrated in the genome with the integrative plasmid pKC796-Tox [23] (Figure 1 step 3). This was the host strain used to express proteins from plasmids selected with this system (Figure 1 step 4).
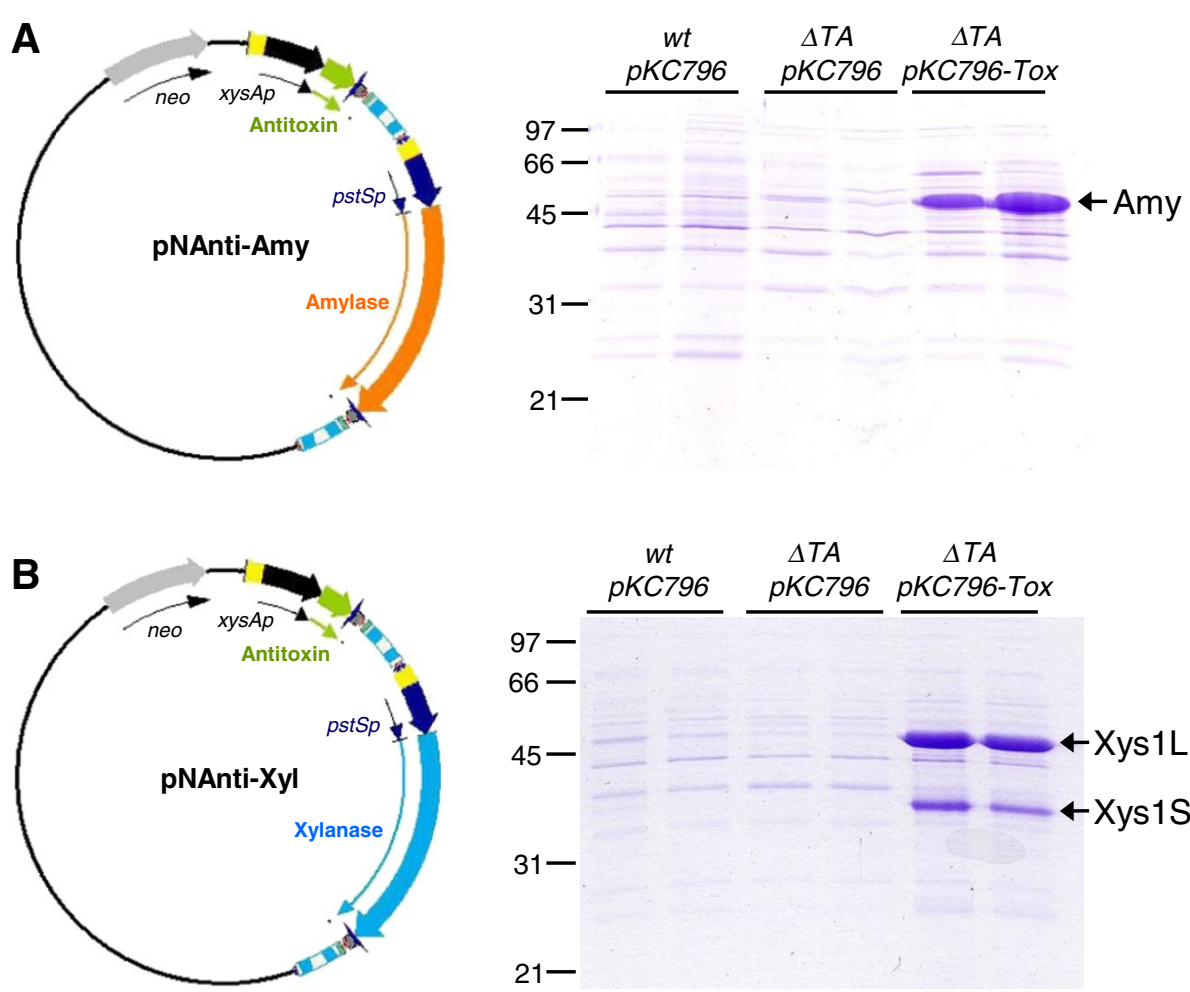

Figure 3 Enzyme production by the different strains. Amylase (A) and Xylanase (B) production by S. lividans wt-pKC796, S. lividans $\triangle T$ TA-pKC796 and S. lividans $\triangle T A-p K C 796-T o x$ transformed with pNAnti-Amy (A) and pNAnti-Xyl (B) after 4 days of culture in YES medium supplemented with $3 \%$ xylose. $10 \mu \mathrm{L}$ of supernatant was loaded in each track. On the left, a diagram of the expression plasmids is shown. 

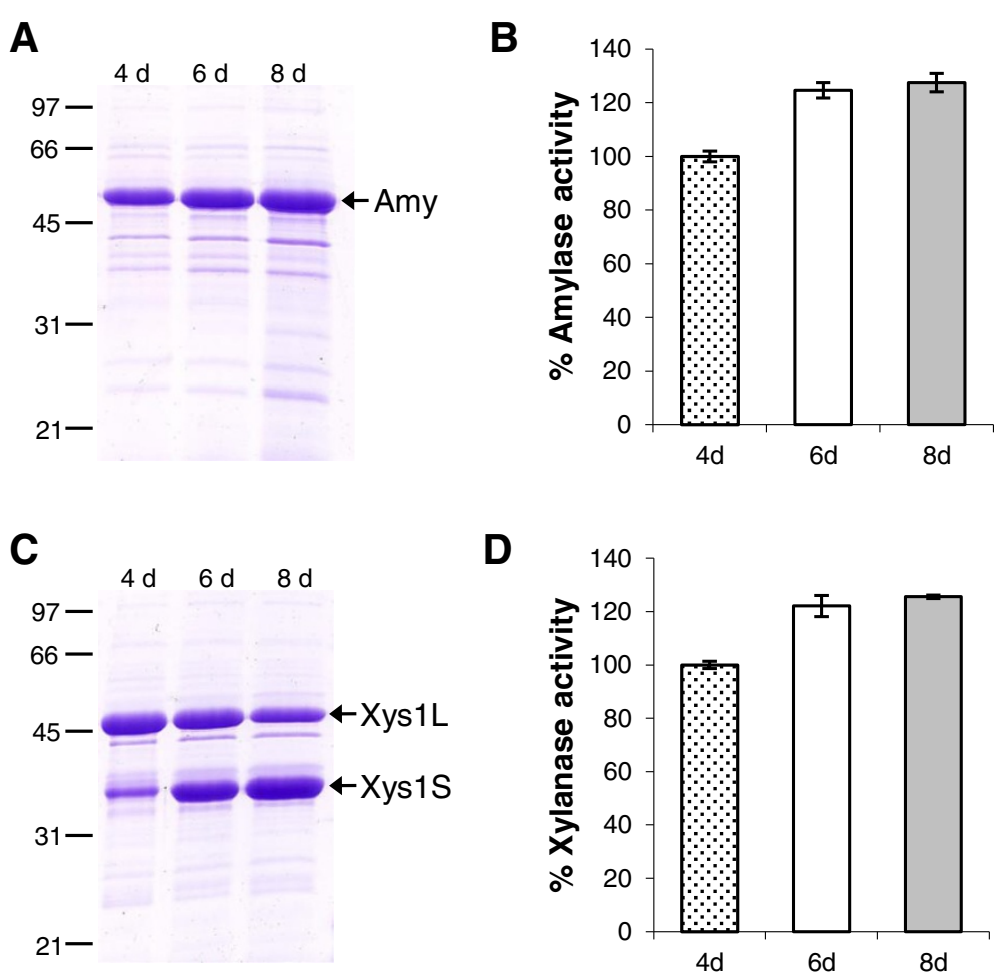

Figure 4 Protein production in long time cultures without selection. Amylase (A) and Xylanase (C) production by S. lividans $\triangle T A-p K C 796-T o x$ transformed with pNAnti-Amy $(\mathbf{A})$ and pNAnti-Xyl (C) after 4, 6 and 8 days of culture in YES medium supplemented with $3 \%$ xylose. $10 \mu \mathrm{L}$ of supernatant was loaded in each track. B and $\mathbf{D}$ show the percentage of amylase (B) and xylanase (D) activity of the supernatants. The histogram bars are the mean of four experiments.

\section{Host strain construction}

S. lividans $\triangle T A$ was obtained by means of REDIRECT technology, as previously described [23]. S. lividans $\triangle T A$ protoplasts were transformed with the multicopy temperature-sensitive thiostrepton resistance pGM160$\mathrm{YefMsl}^{\text {ts }}$ plasmid [23], which expresses YefMsl (antitoxin) under the control of the strong Streptomyces $x y s A p$ promoter $[15,25]$. The strain thus obtained was

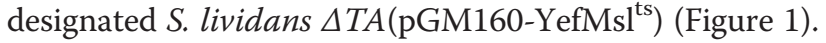
Then, the yoeBsl (toxin) gene was integrated into the genome of this strain with the pKC796-Tox plasmid [23] to yield $\Delta T A-p K C 796-T o x\left(p G M 160-\right.$ YefMst $\left.^{\mathrm{ts}}\right)$. S. lividans $\Delta T A$

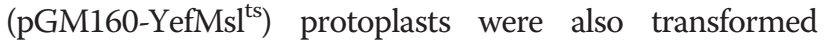
with the empty integrative pKC796 plasmid, generating

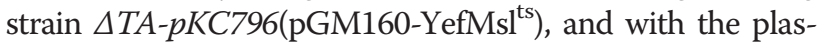
mid containing the complete toxin-antitoxin operon, pKC796-TA, generating strain $\triangle T A-p K C 796-T A$ (pGM160$\mathrm{YefMsl}^{\mathrm{ts}}$ ), which were used as controls. Viability studies of these strains under different temperature conditions (28 and $37^{\circ} \mathrm{C}$ ) demonstrated that the $S$. lividans $\triangle T A$ strain with the toxin gene integrated in the genome $(\triangle T A$ $p K C 796-T o x\left(\mathrm{pGM}_{\left.\left.160-\mathrm{YefMsl}^{\mathrm{ts}}\right)\right)}\right.$ was only viable at $28^{\circ} \mathrm{C}$. It was observed that, when this strain was spread onto R2YE medium and incubated at $37^{\circ} \mathrm{C}$ the cells died because the temperature-sensitive $\mathrm{pGM}^{6} 60-\mathrm{YefMsl}^{\mathrm{ts}}$ plasmid had been lost (Figure 2). However, a few surviving colonies were observed but these were not able to grow when they were reinoculated onto patches on R2YE medium, suggesting an accumulative effect of the toxin (data not shown). This strain was therefore suitable for hosting the expression plasmid, with the antitoxin gene as well as the gene encoding the protein of interest. No toxic effect was observed when plasmid pGM160-YefMsl ${ }^{\text {ts }}$ was lost in the control strains $\Delta T A-p K C 796\left(\mathrm{pGM160-YefMsl}{ }^{\mathrm{ts}}\right.$ ) and $\Delta T A-p K C 796-$ $T A\left(\right.$ pGM160-YefMsl ${ }^{\mathrm{ts}}$ ) when incubated at $37^{\circ} \mathrm{C}$ (Figure 2).

\section{Effectiveness of the yefM/yoeBs/ system as a plasmid stabilization method}

The efficiency of the yefM/yoeBsl system in the maintenance of expression plasmids without antibiotic selection was studied using the $S$. lividans $\triangle T A-p K C 796-T o x$ (pGM160-YefMsl $^{\text {ts }}$ ) strain as host. The production of two proteins - the Amy $\alpha$-amylase from S. griseus IMRU3570 and the Xys1 xylanase from S. halstedii JM8 - was studied in this strain and compared with the one obtained with the $w t-p K C 796$ and $\Delta T A-p K C 796\left(\right.$ pGM160-YefMsl $\left.^{\mathrm{ts}}\right)$ strains, used as controls.

Plasmid pN702Gem3-Anti, a multicopy Streptomyces plasmid that harbours the yefMsl gene under the control of the xylanase promoter $x y s A p$ (see Methods), was used 
to make the expression plasmids by cloning the genes encoding the Amy $\alpha$-amylase or the Xys1 xylanase under the control of the pstSp promoter (see Methods).

Two plasmids, pNAnti-Amy and pNAnti-Xyl, were generated (see methods and Figure 3 ) and were used to transform the S. lividans $\triangle T A-p K C 796-T o x\left(p G M 160-\right.$ YefMsl $^{\text {ts }}$ ) strain and the S. lividans wt-pKC796 and S. lividans $\triangle T A-$ $p K C 796\left(\mathrm{pGM} 60-\mathrm{YefMsl}^{\mathrm{ts}}\right)$ control strains.

After removal of the temperature-sensitive plasmid (pGM160-YefMsl ${ }^{\mathrm{ts}}$ ) by incubation at $37^{\circ} \mathrm{C}$ (see Methods), the transformants of S. lividans $\triangle T A-p K C 796-T o x$ (pNAntiAmy), S. lividans $\triangle T A-p K C 796-T o x(p N A n t i-X y l)$ and the corresponding control strains obtained were grown without antibiotics at $28^{\circ} \mathrm{C}$ in liquid YES medium supplemented with 3\% xylose (an inducer for $x y s A$ and pstS promoters). The amount of amylase and xylanase in the supernatants was analyzed by SDS-PAGE (Figure 3).

After 4 days of culture, strain $\triangle T A-p K C 796-T o x$ produced high levels of amylase (pNAnti-Amy) and xylanase (pNAnti-Xyl) (Figure 3A and B). However, no protein production was observed in the control strains (wt-pKC796 and $\triangle T A-p K C 796)$ transformed with the same plasmids. These results indicated that the absence of antibiotic in the culture medium was responsible for the loss of the expression plasmids (pNAnti-Amy or pNAnti-Xyl) in the control strains that did not harbour the toxin in their genomes. On the contrary, the strain with the toxin gene integrated in the genome needed the presence of the expression plasmid (in reality, the antitoxin gene present in it) to live. Thus, even without antibiotics, this strain was able to maintain the plasmids and, as consequence, produced high levels of proteins.

These results show that the separate-component-stabilization strategy described in this work allows the overproduction of proteins without the addition of antibiotics during the production step.

\section{Plasmid stability}

Plasmid stability without antibiotic selective pressure was evaluated by analyzing the variations in protein production under three different conditions: 1) long-term cultures, 2) after freezing the mycelium and generating new

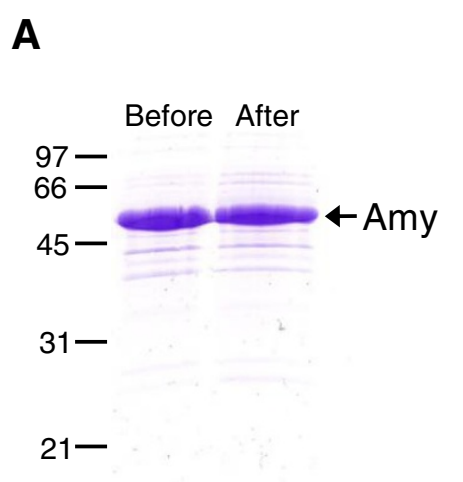

B
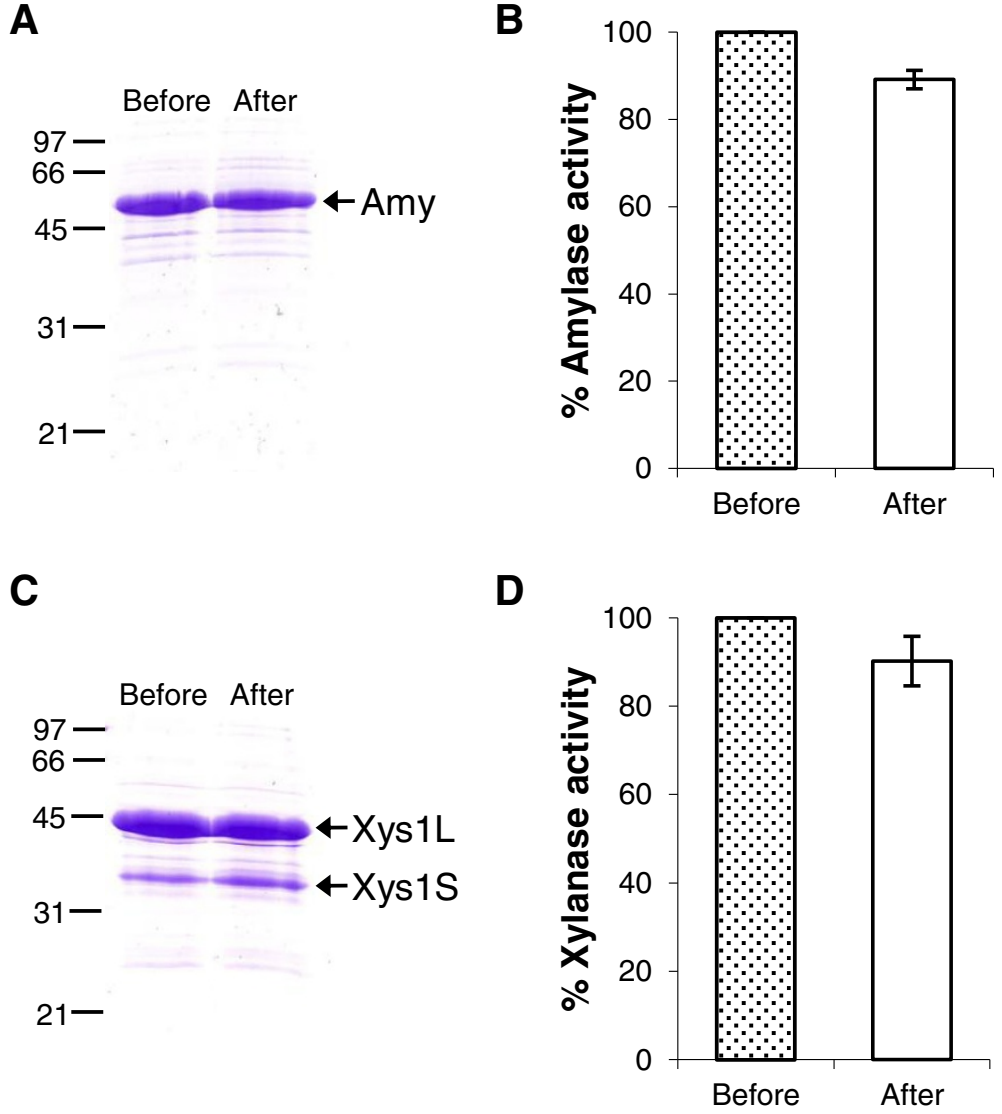

Figure 5 Protein production after a freezing step of the mycelium. Amylase (A) and Xylanase (C) production by S. lividans $\triangle$ TA-pKC796-Tox transformed with pNAnti-Amy (A) and pNAnti-Xyl (C) before and after freezing the mycelium. $10 \mu \mathrm{L}$ of 4-day supernatants was loaded in each track. $\mathbf{B}$ and $\mathbf{D}$ show the percentages of amylase $(\mathbf{B})$ and xylanase $(\mathbf{D})$ activity of the supernatants. The histogram bars are the mean of four experiments. 

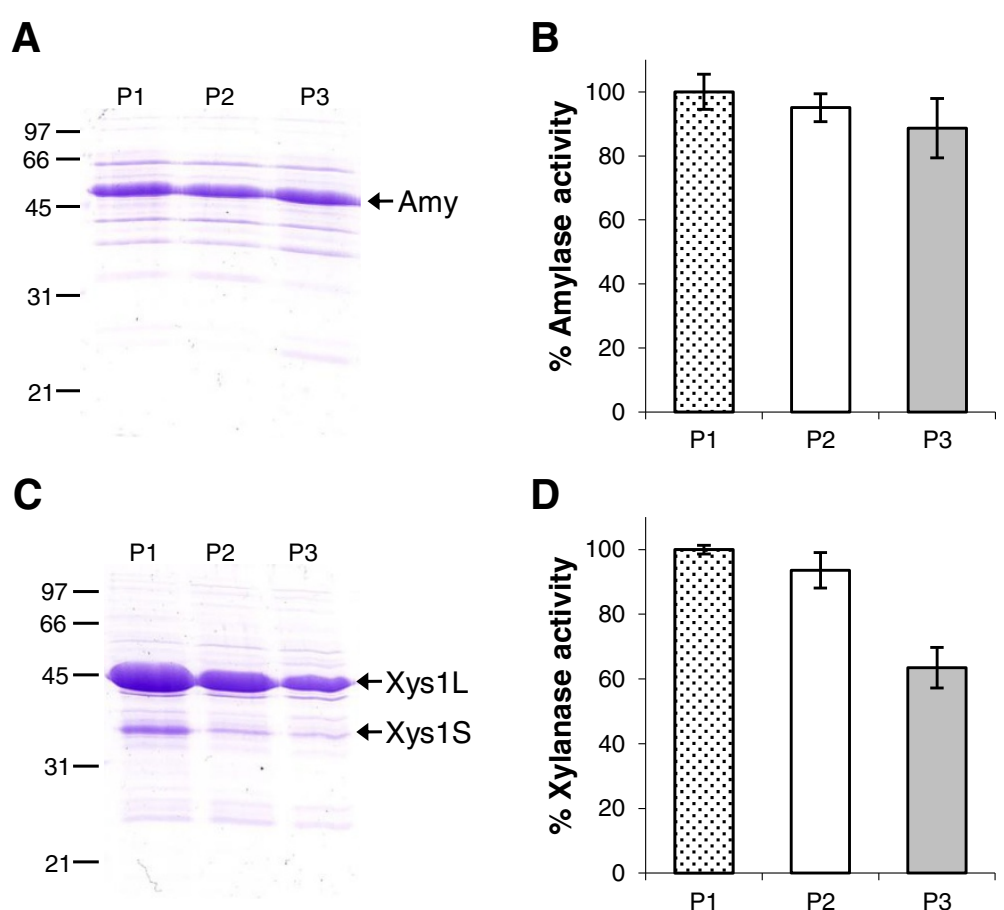

Figure 6 Protein production after serial subcultures. Amylase (A) and Xylanase (C) production by S. lividans $\triangle$ TA-pKC796-Tox transformed with pNAnti-Amy (A) and pNAnti-Xyl (C) after three serial subcultures every two days (P1, P2, and P3) in fresh YES medium supplemented with 3\% xylose. $10 \mu \mathrm{L}$ of 4-day supernatants was loaded in each track. B and $\mathbf{D}$ show the percentage of amylase (B) and xylanase (D) activity of the supernatants. The histogram bars are the mean of four experiments.

colonies for use as inoculum and 3) after performing serial liquid cultures.

The amount of protein produced in long-term cultures was evaluated by SDS-PAGE and by assaying the enzymatic activities in the supernatants of S. lividans $\triangle T A$ pKC796-Tox transformed with pNAnti-Amy or with pNAnti-Xyl. High amounts of both proteins were observed even after 8 days of culture (Figure 4), suggesting that the yefM/yoeBsl system allows stable maintenance of the plasmids that express the amylase or the xylanase in long-term cultures.

The stability of these plasmids after freezing the mycelium was studied by freezing two-day-old liquid cultures of S. lividans $\triangle T A-p K C 796-T o x$ transformed with pNAntiAmy or with pNAnti-Xyl at $-80^{\circ} \mathrm{C}$ in $20 \%$ glycerol. Frozen cells were streaked out for single colonies on R2YE medium without antibiotics, which were then reinoculated on patches on new R2YE plates without antibiotics and used to inoculate liquid YES medium with 3\% xylose. After 4 days of culture at $28^{\circ} \mathrm{C}$, amylase and xylanase production was analyzed by SDS-PAGE and measuring the enzymatic activity of culture supernatants. The yield obtained was compared with that achieved with the original 4-day-culture transformants (Figure 5). Over 90\% of amylase and xylanase activity persisted when the cultures were set up from frozen mycelium (Figure 5B and D).

Table 1 Oligonucleotides used

\begin{tabular}{|c|c|c|}
\hline Name & Sequence $5^{\prime}-3^{\prime}$ & Use \\
\hline LS-005 & TTITTCATATGTCCATCACCGCCAGCGAAG & $\begin{array}{l}\text { Forward oligonucleotide to amplify yefMsl. } \\
\text { The sequence recognized by Ndel is underlined. }\end{array}$ \\
\hline LS-022 & TITITCTCGAGCGCCCGCTCCGCGTCCGGG & $\begin{array}{l}\text { Reverse oligonucleotide to amplify yefMsl. } \\
\text { The sequence recognized by Xhol is underlined. }\end{array}$ \\
\hline MRG-11 & TIITTCATATGGCCCGCAGACTCCGCACC & $\begin{array}{l}\text { Forward oligonucleotide to amplify the amylase gene (amy) from S. griseus. } \\
\text { The sequence recognized by Ndel is underlined. }\end{array}$ \\
\hline MRG-12 & TTITTCTCGAGGCCGCGCCAGGTGTCGTTGAG & $\begin{array}{l}\text { Reverse oligonucleotide to amplify the amylase gene (amy) from S. griseus. } \\
\text { The sequence recognized by Xhol is underlined. }\end{array}$ \\
\hline
\end{tabular}


Table 2 Plasmids used

\begin{tabular}{|c|c|c|}
\hline Plasmid & Characteristics & Reference \\
\hline pGM160 & E.coli/Streptomyces shuttle vector. Thiostrepton and gentamicin resistance. & [24] \\
\hline pGM160-YefMsl & pGM160 derivative. The xysA promoter from S. halstedii controls yefMsl expression. & [23] \\
\hline pKC796 & E.coli/Streptomyces shuttle vector. Apramycin resistance. Integrative plasmid. & [30] \\
\hline pKC796-Tox & pKC796 derivative. The xysA promoter from S. halstedii controls toxin expression. & [23] \\
\hline pKC796-TA & pKC796 derivative. The xysA promoter from S. halstedii controls TA expression. & [23] \\
\hline pXHis1 & pBluescript SK derivative. Ampicillin resistance. The xysA promoter from S. halstedii controls xys 14 expression. & [28] \\
\hline pXHis1-Anti & pXHis1 derivative. The xysA promoter from S. halstedii controls antitoxin expression. & This work \\
\hline pN702Gem3 & E.coli/Streptomyces shuttle vector. Neomycin resistance. High-copy number. & [29] \\
\hline pN702Gem3-Anti & pN702Gem3 derivative. The xysA promoter from S. halstedii controls antitoxin expression. & This work \\
\hline pNUF5 & pN702Gem3 derivative. The pstS promoter from S. lividans controls xylanase expression. & [31] \\
\hline pNUF-Amy & pNUF5 derivative. The pstS promoter from S. lividans controls amylase expression. & This work \\
\hline pNAnti-Xyl & $\begin{array}{l}\text { pN702Gem3-Anti derivative. The xysA promoter from S. halstedii controls antitoxin expression and the pstS promoter } \\
\text { from S. lividans controls xylanase expression. }\end{array}$ & This work \\
\hline pNAnti-Amy & $\begin{array}{l}\text { pN702Gem3-Anti derivative. The xysA promoter from S. halstedii controls antitoxin expression and the pstS promoter } \\
\text { from S. lividans controls amylase expression. }\end{array}$ & This work \\
\hline
\end{tabular}

Finally, plasmid stability in the absence of antibiotic selection was assessed by serial 100-fold dilutions of cultures in fresh YES medium every 48 hours over 6 days. The amylase and xylanase activities of each subculture were analyzed in the supernatants and compared with the enzymatic activity obtained in the original culture (Figure 6). The amylase activity obtained after three rounds of subculture was $90 \%$ of the original culture activity (Figure 6B). Xylanase production was also high throughout the experiment but a loss of xylanase activity was observed in the third subculture, where it was $70 \%$ of that obtained in the original culture (Figure 6D).

These data show that the toxicity of the product of the integrated toxin gene (yoeBsl) in the genome of the host strain (S. lividans $\triangle T A-p K C 796-T o x(p N A n t i-" p r o t "))$ is able to allow stable maintenance of the multicopy expression plasmids carrying the antitoxin gene (yefMsl) and the gene encoding the protein of interest (pNAnti-"prot").

\section{Conclusions}

In this work, we report a new method that allows the production of high levels of proteins in Streptomyces without the use of antibiotics during the production step. This new system is based on the separation of the toxin gene (localized in the genome) and antitoxin gene (localized in the expression plasmid) of the yefM/yoeBsl operon from S. lividans.

The system was found to be useful for the production of high yields of two proteins and could be a powerful tool for producing other proteins of interest in Streptomyces without the drawbacks associated with the use of antibiotics in the production step. This work constitutes the first application of a toxin-antitoxin system to select and stabilize plasmids in Streptomyces.

\section{Methods}

Bacterial strains and growth conditions

The E. coli DH5 $\alpha$ strain [26] was used for the cloning and isolation of plasmids. All strains were grown in Luria-Bertani (LB) liquid broth or on LB agar. All manipulations in $E$. coli were performed following standard procedures [26].

Protoplasts of $S$. lividans 1326 and S. lividans $\triangle T A$ ( $\triangle$ yefM/yoeBsl) were grown on solid R2YE medium after transformation [27]. MSA medium was used for Streptomyces sporulation [27], and liquid YES medium (1\% yeast extract, $10.3 \%$ sucrose $\mathrm{pH} 7.2,5 \mathrm{mM} \mathrm{MgCl}_{2}$ ), supplemented with $0.5 \%$ glucose and $0.5 \%$ glycine for collecting cells to generate protoplasts, and YES medium supplemented with $3 \%$ xylose for protein expression were used. Liquid cultures were carried out in baffled flasks at $28^{\circ} \mathrm{C}$ and $200 \mathrm{rpm}$. All manipulations in Streptomyces were done as indicated by Kieser [27].

\section{Transformation of host strain and colony selection}

Protoplast of S. lividans $\triangle T A-p K C 796-T o x(p G M 160-$ YefMsl) and the control strains, S. lividans wt-pKC796 and $S$. lividans $\triangle T A-p K C 796(\mathrm{pGM160-YefMsl)}$ were transformed with the expression plasmids. After transformation, protoplasts were regenerated $\mathrm{o} / \mathrm{n}$ at $28^{\circ} \mathrm{C}$, then overlayed with $50 \mu \mathrm{g} / \mathrm{mL}$ neomycin to select the cells transformed with the expression plasmids and transferred to $37^{\circ} \mathrm{C}$ for 3 days to eliminate the temperaturesensitive plasmid (pGM160-YefMsl). The loss of the temperature-sensitive plasmid (pGM160-YefMsl) in these transformants colonies was checked by replica-plating the clones onto R2YE plates with $10 \mu \mathrm{g} / \mathrm{mL}$ thiostrepton. Finally, the clones were reinoculated on patches on R2YE plates without antibiotics and incubated at $28^{\circ} \mathrm{C}$. 


\section{Plasmid constructions pN702Gem3-Anti}

yefMsl was amplified by PCR from S. lividans 1326 genomic DNA using primers LS-005 and LS-022 (Table 1). The resulting fragment was digested with NdeI and XhoI and ligated into plasmid pXHis1 [28] (Table 2) digested with the same enzymes to obtain plasmid pXHis1-Anti, which was used as an intermediate plasmid. Plasmid pN702Gem3-Anti was obtained by digesting pXHis1-Anti with BglII, purifying the corresponding yefMsl band and ligating it into pN702Gem3 [29] digested with the same enzyme. In this plasmid, the antitoxin (yefMsl) gene is regulated by the $x y s A$ promoter $[15,25]$.

\section{pNAnti-Xyl}

This plasmid contained the ORF of the xylanase $x y s A$ gene from $S$. halstedii under the control of the pstS promoter and the ORF coding for the antitoxin (YefMsl) under the control of the $x y s A$ promoter. The plasmid was originated from the pNUF5 plasmid [31], which contains the xylanase gene regulated by pstSp; pNUF5 was digested with BglII and BamHI, and the xysA gene band was ligated into pN702Gem3-Anti digested with BamHI.

\section{pNUF-Amy}

The amylase gene (amy) from S. griseus IMRU 3570 [32] was amplified by PCR using primers MRG-11 and MRG-12 (Table 1). The resulting fragment was digested with NdeI and XhoI and, in different steps, was introduced into pNUF5 downstream the $p s t S$ promoter.

\section{pNAnti-Amy}

This plasmid contains the ORF of the amylase gene from $S$. griseus under the control of the $p s t S$ promoter and the ORF coding for the antitoxin (YefMsl) under the control of the $x y s A$ promoter. To construct the plasmid, plasmid pNUF-Amy, which contains the amylase gene regulated by pstSp, was digested with BglII and BamHI and the corresponding band was ligated into pN702Gem3-Anti digested with BamHI.

\section{Sequence analyses}

All constructions were sequenced in both strands using a Perkin Elmer ABI Prism 377 DNA sequencer. In silico plasmids were obtained with the Gene Construction Kit software (GCK, Textco).

\section{Protein analyses}

Protein profiles were analyzed by denaturing polyacrylamide gel electrophoresis (SDS-PAGE) in a MiniProtean II system (Bio-Rad). Proteins were detected by $0.5 \%$ Coomassie brilliant blue $\mathrm{R}$ staining and low-molecular weight standards from Bio-Rad were used as markers.

\section{Xylanase and amylase activities assays}

Xylanase and amylase activities were measured with the dinitrosalicylic acid (DNS) method, using xylose or maltose as standards respectively [33]. One unit of xylanase or amylase activity was defined as the amount of enzyme required to release $1 \mu \mathrm{mol}$ of reducing sugars in one minute (expressed as xylose or maltose equivalents respectively). All data shown are means of at least three different experiments.

\section{Competing interests}

The authors declare that they do not have competing interests.

\section{Authors' contributions}

LS performed most of the experimental work and wrote the manuscript; MD and $\mathrm{RS}$ designed the experiments, directed the work, and wrote the manuscript. All authors have read and approved the final version of the manuscript.

\section{Acknowledgements}

Research in our lab is supported by the grants EUI2008-03631 and BFU2010 17551 from the Ministerio de Ciencia e Innovación to R. Santamaría and M. Díaz respectively and by the grant CSI099A12-1 from the Junta de Castilla y León to R. Santamaría. We thank Dr. G. Muth for the generous gift of plasmid pGM160. MJ Jiménez Rufo is thanked for her excellent technical work and N. Skinner for supervising the English version of the manuscript. The authors also thank the CSIC for the co-financing of this publication in Open Access. The IBFG acknowledges the institutional support granted by the Ramón Areces Foundation during 2011-2012.

Received: 12 February 2013 Accepted: 22 April 2013 Published: 25 April 2013

\section{References}

1. Vrancken K, Anne J: Secretory production of recombinant proteins by Streptomyces. Future Microbiol 2009, 4:181-188.

2. Nakashima N, Mitani Y, Tamura T: Actinomycetes as host cells for production of recombinant proteins. Microb Cell Fact 2005, 4:7.

3. Billman-Jacobe $\mathrm{H}$ : Expression in bacteria other than Escherichia coli. Curr Opin Biotechnol 1996, 7:500-504.

4. Chen R: Bacterial expression systems for recombinant protein production: E. coli and beyond. Biotechnol Adv 2012, 30:1102-1107.

5. Kashima $Y$, Udaka S: High-level production of hyperthermophilic cellulase in the Bacillus brevis expression and secretion system. Biosci Biotechnol Biochem 2004, 68:235-237.

6. Lam KH, Chow KC, Wong WK: Construction of an efficient Bacillus subtilis system for extracellular production of heterologous proteins. J Biotechnol 1998, 63:167-177.

7. Le Loir Y, Azevedo V, Oliveira SC, Freitas DA, Miyoshi A, Bermudez-Humaran LG, Nouaille S, Ribeiro LA, Leclercq S, Gabriel JE, et al: Protein secretion in Lactococcus lactis : an efficient way to increase the overall heterologous protein production. Microb Cell Fact 2005, 4:2

8. Pohl S, Harwood CR: Heterologous protein secretion by Bacillus species from the cradle to the grave. Adv Appl Microbiol 2010, 73:1-25.

9. Brooks SA: Appropriate glycosylation of recombinant proteins for human use: implications of choice of expression system. Mol Biotechnol 2004 28:241-255.

10. Li H, Sethuraman N, Stadheim TA, Zha D, Prinz B, Ballew N, Bobrowicz P, Choi BK, Cook WJ, Cukan M, et al: Optimization of humanized IgGs in glycoengineered Pichia pastoris. Nat Biotechnol 2006, 24:210-215.

11. Anne J, Maldonado B, Van Impe J, Van Mellaert L, Bernaerts K: Recombinant protein production and streptomycetes. J Biotechnol 2012, 158:159-167.

12. Diaz M, Ferreras E, Moreno R, Yepes A, Berenguer J, Santamaria R: Highlevel overproduction of Thermus enzymes in Streptomyces lividans. Appl Microbiol Biotechnol 2008, 79:1001-1008.

13. Harwood CR, Cranenburgh R: Bacillus protein secretion: an unfolding story. Trends Microbiol 2008, 16:73-79.

14. Westers L, Westers H, Quax WJ: Bacillus subtilis as cell factory for pharmaceutical proteins: a biotechnological approach to optimize the host organism. Biochim Biophys Acta 2004, 1694:299-310. 
15. Rodríguez S, Santamaría Rl, Fernández-Ábalos JM, Díaz M: Identification of the sequences involved in the glucose-repressed transcription of the Streptomyces halstedii JM8 xysA promoter. Gene 2005, 351:1-9.

16. Diaz M, Adham SA, Ramon D, Gil JA, Santamaria Rl: Streptomyces lividans and Brevibacterium lactofermentum as heterologous hosts for the production of X22 xylanase from Aspergillus nidulans. Appl Microbiol Biotechnol 2004, 65:401-406.

17. Stieber D, Gabant P, Szpirer C: The art of selective killing: plasmid toxin/ antitoxin systems and their technological applications. Biotechniques 2008, 45:344-346.

18. Szpirer CY, Milinkovitch MC: Separate-component-stabilization system for protein and DNA production without the use of antibiotics. Biotechniques 2005, 38:775-781.

19. Bukowski M, Rojowska A, Wladyka B: Prokaryotic toxin-antitoxin systems-the role in bacterial physiology and application in molecular biology. Acta Biochim Pol 2011, 58:1-9.

20. Peubez I, Chaudet N, Mignon C, Hild G, Husson S, Courtois V, De Luca K, Speck D, Sodoyer R: Antibiotic-free selection in E. coli: new considerations for optimal design and improved production. Microb Cell Fact 2010, 9:65.

21. Pecota DC, Kim CS, Wu K, Gerdes K, Wood TK: Combining the hok/sok, parDE, and pnd postsegregational killer loci to enhance plasmid stability. Appl Environ Microbiol 1997, 63:1917-1924.

22. Shao Y, Harrison EM, Bi D, Tai C, He X, Ou HY, Rajakumar K, Deng Z: TADB: a web-based resource for Type 2 toxin-antitoxin loci in bacteria and archaea. Nucleic Acids Res 2011, 39:D606-611.

23. Sevillano L, Diaz M, Yamaguchi Y, Inouye M, Santamaria RI: Identification of the first functional toxin-antitoxin system in Streptomyces. PLOS One 2012, 7:e32977.

24. Muth G, NuBbaumer B, Wohlleben W, Püler A: A vector system with temperature-sensitive replication for gene disruption and mutational cloning in Streptomyces. Mol Gen Genet 1989, 219:341-348.

25. Ruiz-Arribas A, Sanchez P, Calvete JJ, Raida M, Fernandez-Abalos JM, Santamaria Rl: Analysis of $x y s A$, a gene from Streptomyces halstedii JM8 that encodes a 45-kilodalton modular xylanase, Xys1. Appl Environ Microbiol 1997, 63:2983-2988.

26. Hanahan D: Studies on transformation of Escherichia coli with plasmids. J Mol Biol 1983, 166:557-580.

27. Kieser T, Hopwood DA, Bibb JM, Chater KF, Buttner MJ: Practical Streptomyces genetics. Norwich, UK: John Innes Foundation; 2000.

28. Adham SA, Campelo AB, Ramos A, Gil JA: Construction of a xylanaseproducing strain of Brevibacterium lactofermentum by stable integration of an engineered xysA gene from Streptomyces halstedii JM8. Appl Environ Microbiol 2001, 67:5425-5430

29. Fernández-Abalos JM, Reviejo V, Díaz M, Rodríguez S, Leal F, Santamaría Rl: Posttranslational processing of the xylanase Xys $1 \mathrm{~L}$ from Streptomyces halstedii JM8 is carried out by secreted serine proteases. Microbiology 2003, 149:1623-1632.

30. Kuhstoss S, Richardson MA, Rao RN: Plasmid cloning vectors that integrate site-specifically in Streptomyces spp. Gene 1991, 97:143-146.

31. Diaz M, Esteban A, Fernandez-Abalos JM, Santamaria Rl: The high-affinity phosphate-binding protein PstS is accumulated under high fructose concentrations and mutation of the corresponding gene affects differentiation in Streptomyces lividans. Microbiology 2005, 151:2583-2592.

32. Vigal T, Gil JA, Daza A, García-González MD, Villadas P, Martín JF: Effects of replacementof promoters and modification of the leader peptide region of the amy gene of Streptomyces griseus on synthesis and secretion of a-amylase by Streptomyces lividans. Mol Gen Genet 1991, 231:88-96.

33. Biely P, Mislovicova D, Toman R: Soluble chromogenic substrates for the assay of endo-1,4-ß-xylanases and endo-1,4-ß-glucanases. Anal Biochem 1985, 144:142-146.

\section{doi:10.1186/1475-2859-12-39}

Cite this article as: Sevillano et al: Stable expression plasmids for Streptomyces based on a toxin-antitoxin system. Microbial Cell Factories 2013 12:39.

\section{Submit your next manuscript to BioMed Central and take full advantage of:}

- Convenient online submission

- Thorough peer review

- No space constraints or color figure charges

- Immediate publication on acceptance

- Inclusion in PubMed, CAS, Scopus and Google Scholar

- Research which is freely available for redistribution

Submit your manuscript at www.biomedcentral.com/submit
( Biomed Central 